\title{
THE POSTERIOR STIGMATA OF DIPTEROUS LARVAE AS A DIAGNOSTIC CHARACTER: WITH ESPECIAL REFERENCE TO THE LARVAE INCRIMINATED IN CASES OF MYIASIS.
}

By MALCOLM EVAN MACGREGOR, B.A. (Cantab.), F.R.M.S., Carnegie Student in Medical Entomology.

(From the Bussey Institution, Forest Hills, Boston, Mass.)

(With Plates XIV-XVI and 3 Textıfigures.)

DURING investigations at the Bussey Institution on the rôle played by certain dipterous larvae in experimentally induced myiasis, with the object if possible of clearing a little of the doubt with which so much of the question is shrouded, the need of good diagnostic characters for these larvae was brought forcibly to my attention.

This need is one that is felt both by entomologists and physicians, since, when cases of myiasis are met with, it is of the greatest importance that the species of fly concerned shall be readily determined without the need of rearing the larvae to the adult stage, at best a method that can but seldom be undertaken, owing to the fact that the material that one has to pronounce upon is often dead. Thus at the suggestion of Dr Wheeler and Mr Brues, a careful comparison of the morphological characters of the posterior stigmata was undertaken, and though this cannot claim to be in any way the first of such studies, since there are many good illustrations of the various forms of stigmata occurring in the different larvae, notably in Dr C. Gordon Hewitt's book, The House$f l y$, and Mr Nathan Bank's paper on The structure of certain dipterous larvae, with special reference to those in Human Foods, as well as in the works of other authors, including several old papers; yet I think 
the subject has hardly been given the attention it deserves. I have endeavoured therefore to secure actual photomicrographs of these organs, under the moderately high powers of the microscope, and while these are not in every case quite as clear as some of the other illustrations already mentioned, they are both more accurate and show the remarkable differences more clearly.

\section{Technique.}

The aim has been to obtain a method that will envolve the least difficulty in the determination of any particular larva, and the method of preparing the specimens for identification will therefore first be briefly dealt with. This rests on the every-day principle of removing the whole of the softer tissues by boiling in potash, so as to leave only

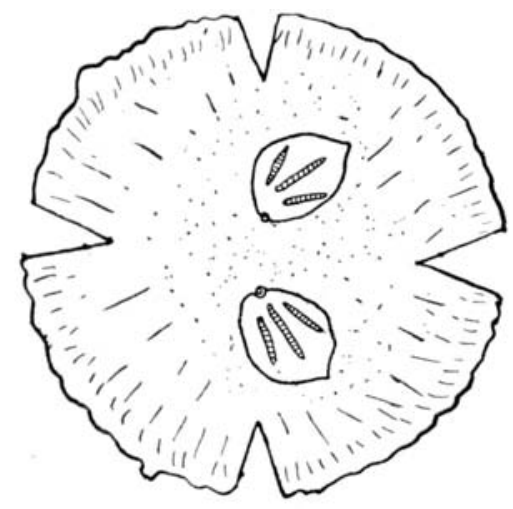

Text-fig. 1. Diagram showing method of splitting the edge of the cuticle in specimens before mounting.

the chitinous structures intact. The larvae are hardened in absolute alcohol for several days, and are then sectioned by hand, without imbedding, with a keen microtome knife or, what does almost better, a carefully sharpened Gillete razor blade. With the larva held between the finger and thumb, posterior end uppermost, about $\frac{1}{32}{ }^{12}$ is cut off, care being taken that the blade passes below the stigmal plates which are seen usually as two little yellowish-brown dots lying on each side of the middle line at the posterior end of the animal. The section is then examined under the low power of the microscope, to ascertain that the stigmata have suffered no injury, and if possible when the section is large enough the periphery of the cut edge of the cuticle is carefully split by slight incisions with the razor at four points at right angles to each other, as shown in Text-fig. 1. 
The next procedure is to put the section in $10 \% \mathrm{KOH}$ solution, which is raised to the boiling-point and kept boiling gently until the specimen is quite free from all attached tissue, and only the chitinous structures remain. After removal from the $\mathrm{KOH}$ solution, it is washed in water thoroughly, dehydrated in the usual way, and cleared in cloveoil. It is then transferred to a drop of balsam on a slide, and arranged with the exterior surfaces of the stigmata upwards. A coverglass is applied, and gentle pressure administered over the object by means of a clip, until the balsam has set, in order to get the object as flat as possible.

The pressure must be most carefully adjusted however, as some specimens are extremely delicate.

\section{The Structure of the Stigmata.}

Owing to the great difficulty of obtaining material in the wintertime, my observations (which in this paper are given merely in preliminary form) are necessarily confined to comparatively few genera, and one is thus unable to predict what degree of variation in form and structure of the stigmata may occur in other genera and species, but there is no reason to suppose that these would not exhibit equally marked variation; as is borne out to some extent by other examples besides those published here, and also by the rough illustrations that, here and there, are to be found scattered through the literature on the anatomy of dipterous larvae.

Of these forms of stigmata there are two main types which may be classed under the following headings, namely the schizotreme-type and ptychotreme-type (see Plate XIV, fig. 1 and Plate XV, fig. 7), and these it would seem, from the material studied, coincide individually with the main taxonomic relationships of the insects: that is, it appears in many cases that related genera have the same main type of stigmata, while the one genus is distinguished from the other by variations in

(1) the orientation of the stigmata with reference to the angle made to the longitudinal axis of the larva ;

(2) in the distance between the stigmata themselves;

or, (3) by variations of the gross structure, and shape of these organs : such as a thickening or otherwise of the chitinous ring that borders them, etc.

The specific differences are to be found in the quite often remarkable variations that occur in the finer structure, i.e. in the transverse bars 
of the slits, both as to arrangement and number, as well as the variation of the position of the "button" (vide infra).

In addition to these two main classes, there are several other forms, which occur particularly in the Oestridae. These forms differ from one another so remarkably that it will be sufficient to describe them individually, as each is quite characteristic of the genus to which it belongs.

\section{The Variability of the Form of the Stigmata.}

In the same genus and species there is apparently remarkably little variation in the position and structure of the posterior stigmata in the larvae (once these have reached the second instar), but the variation in the different genera and species that have been available for study is so marked that the most casual observer would have no difficulty whatever in recognising them.

Under the two headings already mentioned, into which the types of the stigmata may be classed, a brief description of the most salient characters of each will now be given.

\section{The Schizotreme-type of Stigmata.}

The vast majority of the stigmata examined belong to this class. Essentially they constitute two almost circular chitinous plates, with thickened edges, forming distinct rings that enclose the whole organ.

The plates may be arranged at varying distances from one another at the posterior end of the larva, and as nearly as possible on each side of the median sagittal plane; while they may be placed also

(1) above the median horizontal plane,

(2) in this plane, and

(3) below this plane.

Whatever the actual position in this regard, the slits of the schizotreme-type however seem to be invariably three in number but the angle which they make with each other, and with the median sagittal plane, is very often, in the different genera and species, quite specific.

These slits are spanned by delicate chitinous bars, which may pass from one side of the slit to the other, either as simple straight rods, or as rods having one or more branching processes that anastomose with the processes from other rods, and thereby form a network.

In many of the more complicated examples, examined under the microscope, there appear what at first sight might be taken to be nuclei 
at the bases of these rods, but these "nuclei" are merely the optical effect produced by the intersection of rods, or processes from them, at another focal plane. In every case the slits are very much longer than they are broad; are usually all about the same length (although in some instances the second slit is somewhat longer than the other two); and the extremities are always rounded off.

In most larvae, at the inner side of the circumference of the plate, or, as is the case generally, situated on the ring, there is what has been previously referred to as the "button," a term used by Mr Banks. This structure, so far as I have been able to determine, is the place of attachment of muscles. It is not however invariably present in the slit class of stigmata, but its exact position, when it does occur, varies in different species, and is, when taken in conjunction with others, one of the characters on which the differentiation of the larvae can be based.

The thickened periphery of the stigmal plate, which has been called the "ring," is sometimes incomplete, and when this is so it is at the region where the "button" ought to be situated that this break often occurs. An example of such a condition is to be found in the Sarcophagidae. The significance of this condition $I$ have not been able to discover, but it is a valuable character in the work of diagnosis, since only two examples of this arrangement have yet been found, namely in the Sarcophagidae and in Chrysomyia.

\section{The Ptychotreme-type of Stigmata.}

Examples of this type are somewhat less numerous than those of the preceding type, and while for the most part the arrangement is to a great extent similar to those just described, yet in many details they are entirely unlike. The "ring" is usually very well marked, but sometimes the chitin of the plate is so dense that the inner margin of the "ring" is difficult to distinguish. Within the "ring" however there is arranged a chain, convoluted to a varying degree, and with remarkably different configuration according to the different species. It may be either in the form of an unbroken chain, or broken into segments, as in Plate XV, fig. 8 ; but in this class are included only those forms of stigmata which have a distinct convoluted chain, of one kind or another, within the outer chitinous ring; and to decide on this point as to whether a particular type does, or does not, belong to this class has never been found a matter about which there could be much doubt. The typical examples of the class here illustrated are those of the house-fly (Musca 
domesica), and the stable-fly (Stomoxys calcitrans). Nevertheless some care has to be taken in recognising one or two stigmata of the ptychotreme-type, for although the convoluted chain is always present its recognition may be rendered difficult by the chitin of the "ring" having become so extended that it lies as a plate below, leaving spaces only at intervals where the course of the chain can be seen. A case of this sort is met with in the stigmata of the horn-fly (Haematobia serrata). However, by careful focussing there is actually no difficulty in recognising the chain even in this case, especially when the attention has once been drawn to this not very commonly occurring condition.

In the ptychotreme-type of stigmata the " button" is always present, and usually very distinct, and in some cases where the stigma is bounded by a very delicate chitinous ring, chitinous processes often run out to it like irregularly arranged spokes in a wheel, and no doubt serve as supporting pieces.

The two classes, schizotreme-type and ptychotreme-type, are no doubt homologous with one another if the latter be regarded as a simple difference leading from a radiant to a convoluted arrangement; and this idea is to a degree supported by the fact that there are usually three breaks in the course of the convoluted chain, as there are also three slits in the other type of stigmata.

\section{Descriptions of the Stigmata illustrated.}

I will now give a brief description of the individual stigmata here illustrated, together with one or two others, beginning with the schizotreme class first. As the typical example of this class the stigmata of Lucilia caesar may be selected.

Lucilia caesar Linn. (Plate XIV, figs. 1 and 2.)

These stigmata in the fully formed larvae occur as two fan-shaped stigmal plates, from $0.44 \mathrm{~mm} .-0.46 \mathrm{~mm}$. in breadth, and lying from $0.40 \mathrm{~mm} .-0.48 \mathrm{~mm}$. apart, when the distance measured is from the centre of one "button" to the centre of the other. The "button" in this genus is well developed, and the " ring" is delicate and sharply delimited from the rest of the plate. The slits are three in number, and are crossed by from 11-14 separate relatively broad bars, which run from the extreme edge of the one side of the slit to the extreme edge of the other. Between these bars the gaps are large, fairly equal in size, and clearly outlined: their longitudinal measurement being usually greater than the breadth of the bars. 
Cynomyia cadaverina Desv. (Plate XIV, figs. 3 and 4.)

The stigmata of this larva appear also as two fan-shaped plates. Although the actual size and shape of each plate, and the distance between them, are almost the same as in Lucilia caesar, there are wide differences which distinguish the two genera. The "ring" in $C$. cadaverina is rather more heavily chitinised, the "button" is larger, and more densely surrounded with chitin, while the angles which the slits make to the median sagittal axis of the larva are very much greater.

Moreover there is an essential difference in the arrangements of the bars which cross the slits. These instead of running from the extreme edge of the one slit to the extreme edge of the other, as in Lucilia caesar, start a little distance from one side of the edge and run over to an equal distance beyond the other edge, tending to arch over the gap to some extent (fig. 4). This arrangement gives a characteristic appearance not found in any of the other genera that have been examined. The breadth of the stigmal plate in this case is from $0.52-0.56 \mathrm{~mm}$. and the distance between the "buttons" from 0.48-0.53 mm.

\section{Calliphora vomitoria Linn. (Plate XV, figs. 5 and 6.)}

In the larva of this fly the stigmata are considerably larger than those of the larvae of the preceding two genera, being from $0.60-0.68 \mathrm{~mm}$. in breadth, with the distance between the "button" of the one plate and the "button" of the other ranging from $0.56 \mathrm{~mm} .-0.64 \mathrm{~mm}$. The " ring" is very heavily chitinised, and the " button " well marked. The angles of the slits are very much the same as in the case of Lucilia caesar, but here again the characteristic difference of the genus is found in the bars that cross them. These are far more numerous than in the other examples described above, and though in this case they run from the extreme edge of the one side to the extreme edge of the other, as in Lucilia caesar, they are nevertheless only about half the breadth, and from 20-24 in number for each slit.

Sarcophaga sarraceniae? Riley. (Text-fig. 2b.)

In the stigmata of this species we meet several remarkably different features. The most striking is perhaps the position of the slits. Instead of making wide angles with one another, they approach a parallel arrangement, and what is still more noticeable, are arranged so that 
they are not far from being parallel also with the median sagittal plane of the larva. The slits themselves are comparatively broad, with the bars arranged at the "extreme edge" position. Of these there are a fairly large number, but their form resembles that found in Lucilia caesar, rather than that of Cynomyia cadaverina.

Another strikingly different feature, and one, as has been already said, that has only been found to be shared by Chrysomyia (sp. incert.), occurs in the "ring." In the stigmata of Sarcophaga sarraceniae this "ring" is especially distinct and delicate, but at the point where in the other specimens the "button" is situated, the "ring" is broken and a gap occurs. Thus in the stigmal plate the "button" is absent altogether, though the broken ends of the "ring" are somewhat
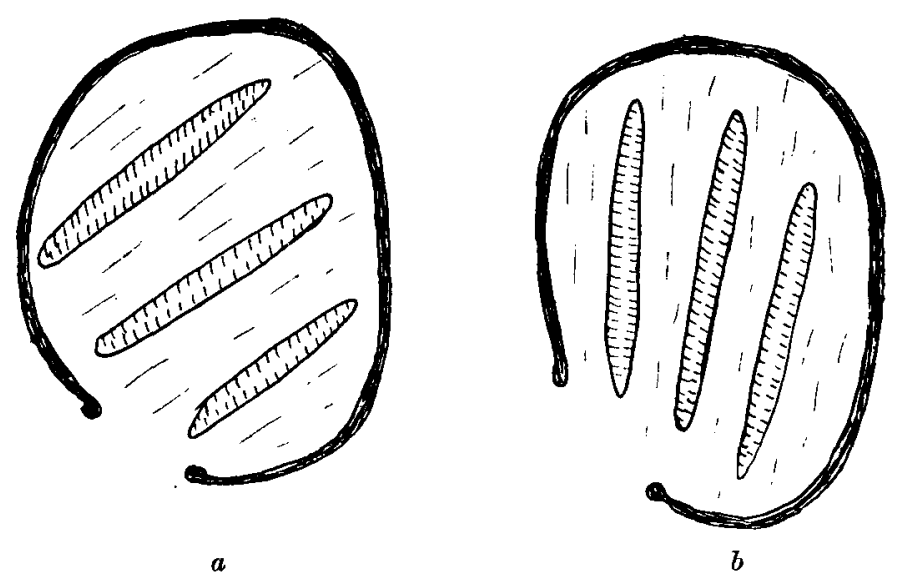

Text.fig. 2. Posterior stigmata of (a) Sarcophaga sarraceniae and (b) Chrysomyia (?). Diagram of one plate of each to show different arrangement of slits.

thickened and rounded. Taking into account first the almost parallel arrangement of the slits, and then this latter feature, the larvae of this fly are very easily recognised, and there is no need to consider also the finer structure in order to differentiate it satisfactorily from all the others except Chrysomyia (?). From this genus it can however be distinguished by the fact that in Chrysomyia (sp. incert.), though the general arrangements of the slits and the "ring" are similar to those of Sarcophaga sarraceniae, in the former the slits, while parallel with one another, are diagonally arranged, and cannot in any way be considered as parallel with the median sagittal plane of the body. 


\section{Chrysomyia sp. incert. (Text-fig. 2a.)}

The general arrangements of all parts of the stigmata are similar to the stigmata of Sarcophaga, but differ in the manner described, i.e. in the angle that the slits make with the median sagittal plane, and in the fact they are obliquely parallel with one another.

\section{The Ptychotreme-type of Stigmata.}

As the typical example of this class, the stigmata of the house-fly (Musca domestica) will be taken. In this class the stigmal plate is rather different from that found in the schizotreme-type, tending to be more circular, or in some cases triangular, than fan-shaped: while the position of the "button" is also different, since when this occurs instead of lying on the "ring" it is situated within it, and usually on a line with the horizontal diameter, either touching the inner side of the " ring," or removed some little distance from it.

\section{Musca domestica Linn. (Plate XV, figs. 7 and 8.)}

In the larva of this fly the "ring" is always very heavily chitinised, and may be as narrow as in the example shown in fig. 7, or it may be very much broader. The periphery of the "ring" slopes forward (i.e. with reference to the anterior end of the larva), so that the stigmata become saucer-shaped, with the concave surface applied to the animal. The exterior surface, which may be regarded as the bottom of the saucer, is flattened, and it is upon this that the convoluted or undulating chain occurs.

This chain consists of a chitinous net-like band, due to a complicated anastomosis of the rods, and is somewhat similar to the arrangement of the rods spanning the slits of the schizotreme-type of stigmata. At first sight, under the low powers of the microscope, the chain appears to be continuous, but on closer observation it is seen to be made up of three sections which are very easily distinguished under the higher magnifications. Taken as a whole the chains of each stigmatic plate have nine complete convolutions in their course; when these are counted by the crest of each wave, but under the high magnifications again, it can be seen that they occur actually as three convolutions to each of the three sections.

Breadth of plates, $0 \cdot 24-0.28 \mathrm{~mm}$.; distance between "buttons" $0 \cdot 25-0.30 \mathrm{~mm}$. 
Stomoxys calcitrans Linn. (Text-fig. $3 b$.

In larvae of this fly the "rings" of the stigmal plates are, roughly, triangular in shape with the adjacent sides straightened and parallel. The "button" is relatively very large, and heavily chitinised, and situated near the middle of the plate. On three sides of the "button," and at right angles to one another, are the three sections of what may be regarded as the broken convoluted chain, with an S-shaped configuration, and very clearly defined. Each section of the chain is crossed throughout its length by a branching bar system similar to that seen in the other types of stigmata, except that in this case the bars are exceedingly slender, and though they can be vaguely made out under the higher powers of the microscope ( $\frac{1}{6}$ objective and $\times 5$ ocular), it needs the very highest combinations ( $\frac{1}{12}$ oil imm. objective and $\times 12$ ocular), to resolve them clearly. Under a magnification of 1440 diams. the bar system can be seen to consist not, as in the stigmata of Lucilia caesar, of a single line of branching rods, but of a series of rods in more than one plane which thus produce a most complicated network, the interstices of which measure only a few microns across. The general appearance of the stigmata of Stomoxys calcitrans nevertheless, even at low magnifications, is quite characteristic, and cannot easily be mistaken for any other. The measurements of the stigmata are as follows: distance between "buttons," 0.22-0.26 mm.; breadth across plate, $0 \cdot 28-0 \cdot 30 \mathrm{~mm}$.

\section{Haematobia serrata Desv. (Text-fig. 3 a.)}

Of all the forms described in this paper this has yielded the least definite anatomical data; for two reasons: first, that the chitinous "ring" and stigmal plate generally are so heavily chitinised, that mounted in the manner described they are almost opaque to transmitted light under the microscope, except at irregular spots; and secondly that owing to scarcity of material I have not been able to make a sufficient comparative study. That this form of stigmata belongs to the ptychotreme-type there can be no question, however, because by careful manipulation and focussing of the microscope the convoluted chain can be traced over its whole length, while it is very clearly seen at the irregular spots mentioned above where the chitin of the plate is not so thick. That the chain lies well above the heavier chitinous structures of the plate, as with the other stigmata belonging to the ptychotreme-type, is particularly well seen in this larva, for it is situated 
at a plane so much higher than these structures that when they are in sharp focus there is not the slightest indication of the chain's presence, and the microscope has to be racked up several revolutions of the fine adjustment before it in turn comes into focus. Here again the convoluted chain is broken into three segments which have a similar position to those of Stomoxys calcitrans, but differ so markedly in the extraordinary arrangement of the convolutions that there is no possibility of mistaking the one for the other. The form of one segment of each is shown in Text-figure $3 a$, and the measurements of the stigmata are : distance between "buttons," $0 \cdot 27-0.28 \mathrm{~mm}$.; breadth of plate, $0 \cdot 23-0 \cdot 25 \mathrm{~mm}$.

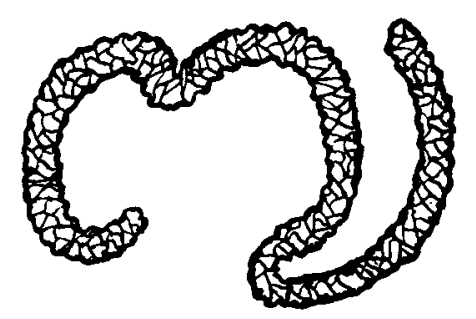

$\boldsymbol{a}$

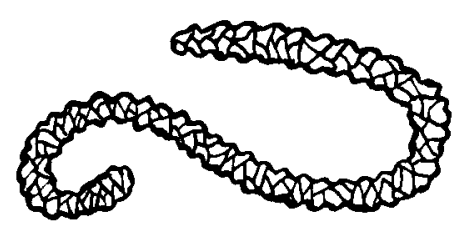

$b$

Text-fig. 3. Diagram showing the difference in the form of one segment of the convoluted chain in the stigmata of (a) Haimatobia serrata and (b) Stomoxys calcitrans respectively.

\section{Gastrophilus equi Clark.}

(Plate XV, figs. 9, 10 and Plate XVI, figs. 11, 12.)

In Gastrophilus equi we meet with a very unusual condition, in that the stigma occurs as a single large plate, which may be regarded as formed by the fusion of the usual two stigmal plates found in those larvae that have been already considered. The fusion may be regarded as having taken place at the top and bottom of each of these two plates, while the parts between have become modified, and hollowed out, so as to leave a gap midway between, into which, one from above and one from below, two tongue-bars project (see fig. 9). On each plate, and running in sinuous parallel arrangement with one another, are three chitinous structures which remind one of the "slits " in the schizotremetype of stigmata, and like these are crossed by bars. These bars, nevertheless, are more widely set apart, and rarely have a tendency to branch in the same way as those of the other types: moreover their finer structure is entirely unlike that found in the previously described 
specimens. It consists, as will be seen in fig. 12, of rods that are thickened at their centre in such a way that a diamond or square-shaped formation results, which is hyaline to some extent and highly refractive, so much so that at the high magnifications that have to be used when examining this structure, it has not been by any means easy to feel certain when its true focus had been attained.

The base on which these sinuous " slits" are placed, and which has been referred to as the "plate," when examined from the exterior surface presents the appearance described above, i.e. an appearance suggesting its possible formation by the fusion of the two separate plates found in the schizotreme- and ptychotreme-types of stigmata: examined from the under surface of the section however, a strikingly beautiful and remarkable structure is seen. Observed in this position, there can be seen, arising at regular intervals on the lower surface of the plate, six to eight chitinous processes, which curving upwards fuse with a small ring directly above the centre at the back of the plate, and thus form, as it were, a gourd-shaped basket. I was fortunate enough to be able to obtain a fairly large number of these larvae alive from the stomach of a horse that was autopsied at the Vaccine Laboratory here, and was in this way permitted to study the stigmata in much greater detail than has been the case with many of the other specimens, in that the physiology as well as the anatomy could receive attention. It would be beyond the purpose of this paper to discuss the former subject here, but it might be interesting to state that the physiology of these organs appears by no means as simple as it has been supposed, and that while the sinuous bar systems, or so-called " slits," have been described as lying on the plate, they are in reality attached to a very delicate transparent membrane which covers it, and in the fresh larvae it has been found possible, under the microscope and using special instruments, to dissect off this membrane, bringing away with it the bar systems intact (see fig. 11). Breadth of stigmal plate, $2 \cdot 6-3 \cdot 1 \mathrm{~mm}$.

Oestris ovis Linn. (Plate XVI, figs. 13 and 14.)

Here again the stigmata are found to be two separate plates, but the conspicuous "ring" is entirely absent. These plates are from 1.20-1.26 mm. in breadth, and the distance from " button " to " button" is from 1.24-1.30 mm. The "buttons" are very well defined, and from them there radiates over the entire surface of the plates a very 
conspicuous dense chitinous network, resembling very much in arrangement the appearance of a mycelium of some fungus growing on culture medium, or the "picture" produced by the cathode electric brushdischarge on a photographic plate. It is a type unlike anything else that has been described, or found in any of the specimens yet examined, and in the Oestridae there seems to be the greatest difference in the form of the stigmata, each genus apparently having its own individual type.

In conclusion I should like to thank Dr Wheeler and Mr Brues for their help and suggestions, which at all times they have been so ready to give ; and Drs Ernst and Wolbach, of the Harvard Medical School, for their courtesy in permitting me to use the most excellent photomicrographic apparatus that they possess, without the use of which I could have made very little of these difficult subjects for photography.

\section{DESCRIPTION OF PLATES XIV-XVI.}

Noтs : magnifications given are only approximate.

Fig. 1. Posterior stigmata of Lucilia caesar, $\times 50$ diams., showing both plates in situ.

Fig. 2. Posterior stigmata of Lucilia caesar, $\times 140$ diams, showing one stigmal plate.

Fig. 3. Posterior stigmata of Cynomyia cadaverina, $\times 50$ diams., showing both plates in situ.

Fig. 4. Posterior stigmata of Cynomyia cadaverina, $\times 150$ diams, showing one stigmal plate.

Fig. 5. Posterior stigmata of Calliphora vomitoria, $\times 40$ diams., showing both plates in situ.

Fig. 6. Posterior atigmata of Calliphora vomitoria, $\times 120$ diams., showing one stigmal plate.

Fig. 7.' Posterior stigmata of Musca domestica, $\times 75$ diams, showing both plates in situ.

Fig. 8. Posterior stigmata of $M$ usca domestica, $\times 225$ diams.; showing one stigmal plate.

Fig. 9. Posterior stigmata of Gastrophilus equi, $\times 30$ diams., showing the single stigma.

Fig. 10. Posterior stigmata of Gastrophilus equi, $\times 60$ diams., showing the bar system in greater detail.

Fig. 11. Posterior stigmata of Gastrophilus equi, $\times 40$ diams., showing the bar system on the membrane.

Fig. 12. Posterior stigmata of Gastrophilus equi, $\times 100$ diams., showing the bar system on the membrane in greater detail.

Fig. 13. Posterior stigmata of Oestris ovis, $\times 30$ diams., showing both plates in situ.

Fig. 14. Posterior stigmata of Oestris ovis, $\times 80$ diams., showing one stigmal plate. 


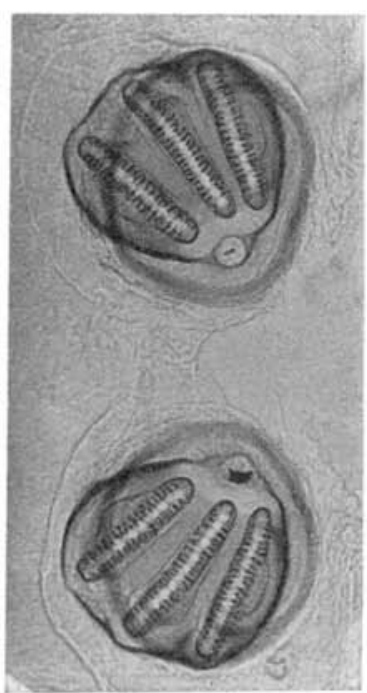

Fig. 1.

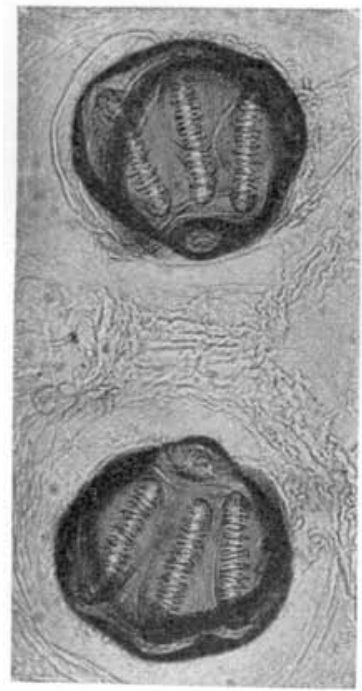

Fig: 3 :

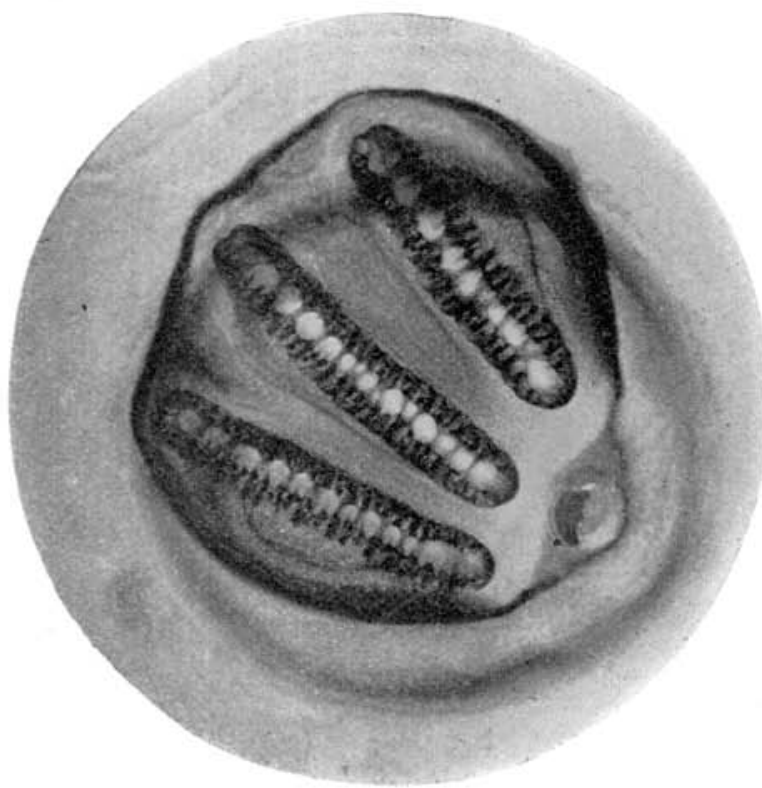

Fig. 2.

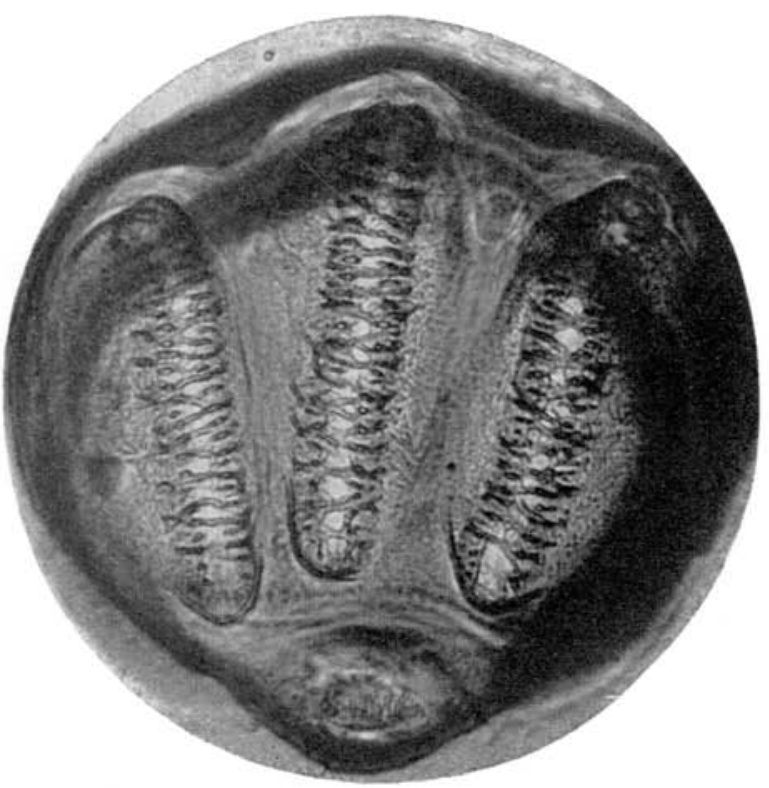

Fig. 4. 
PARASITOLOGY, VOL. VII. NO. 2

PLATE XV

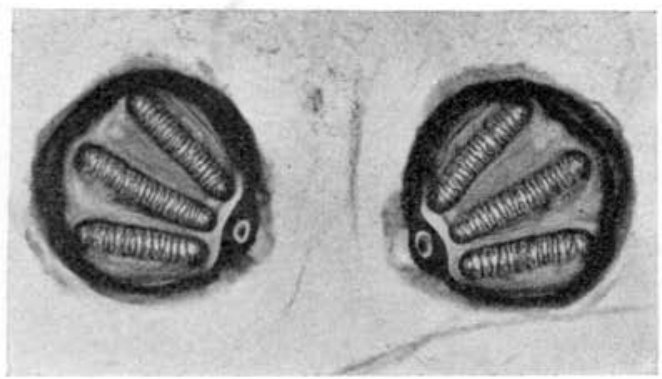

Fig. 5.

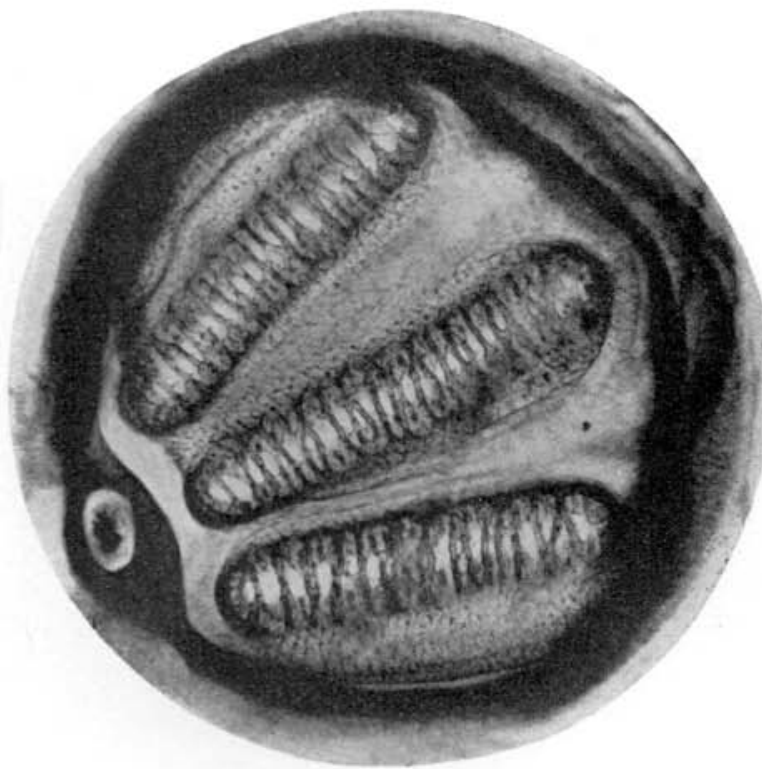

Fig. 6.

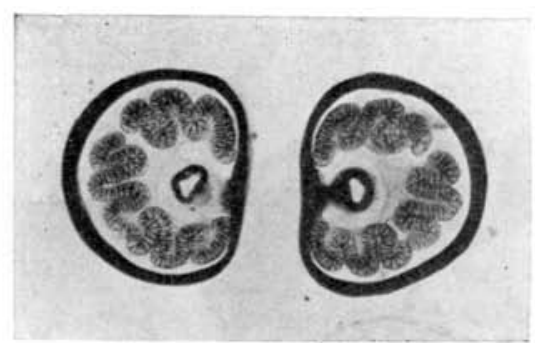

Fig. 7 ,

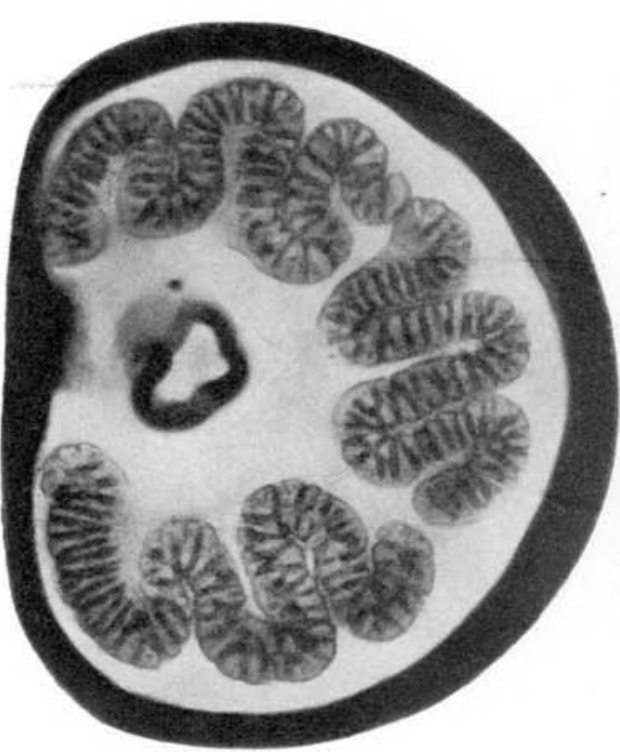

Fig. 8.

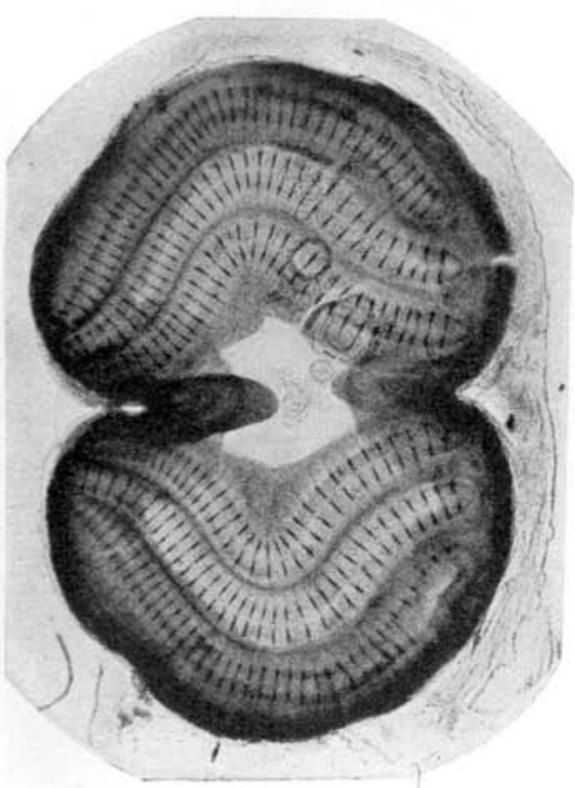

Fig. 9.

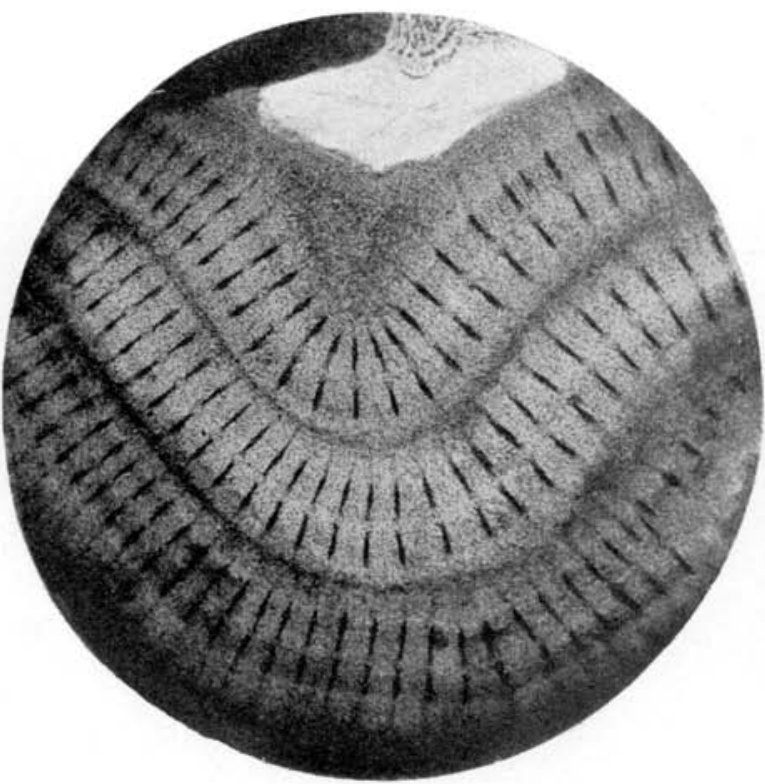

Fig. 10. 
PARASITOLOGY, VOL. VII. NO. 2

PLATE XVI

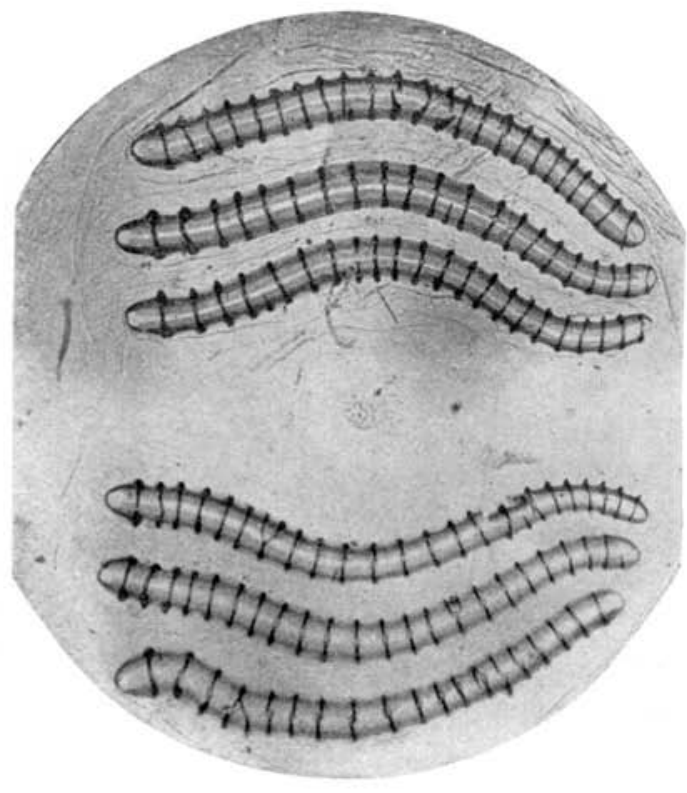

Fig. 11.

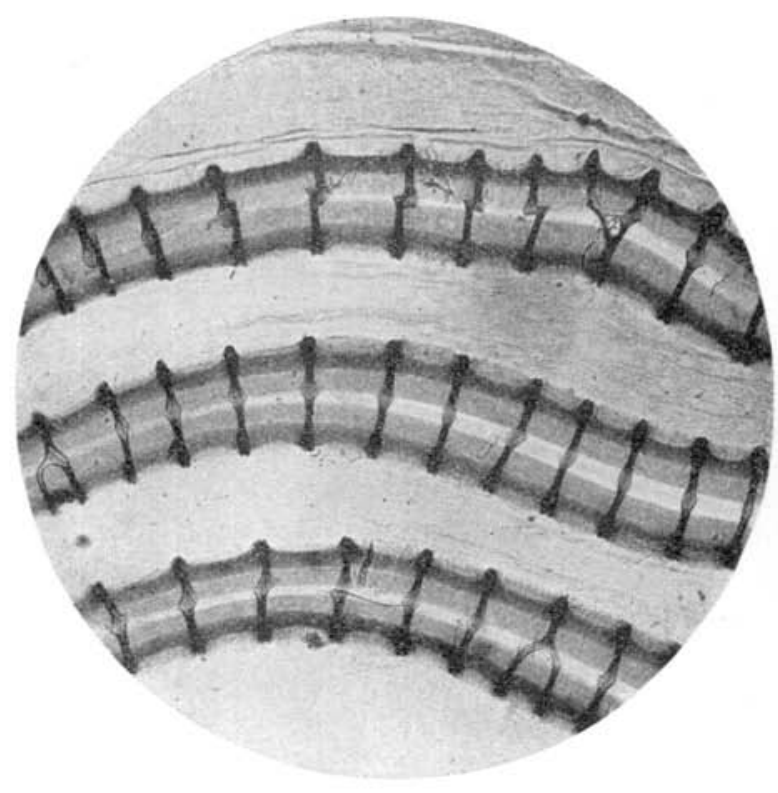

Fig. 12.
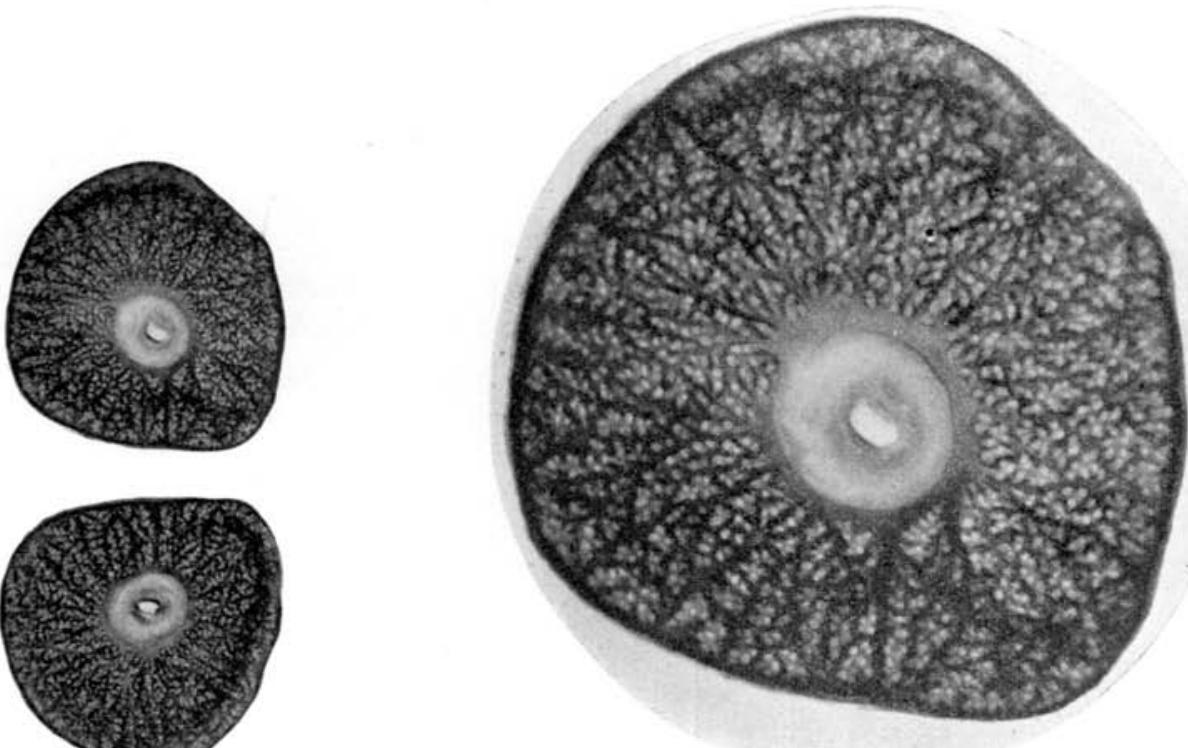

Fig. 13

Fis. 14 